\title{
Anesthetic Considerations in a Case With Massive Recurrent Clivus Chordoma
}



\section{Pardis Soltanpoor ${ }^{\circledR}$, Faranak Behnaz $^{*(\oplus)}$, Hamidreza Azizi Faresani $^{(\oplus}$, Afsoun Seddighi $^{(\oplus}$, Maede Karimian ${ }^{1}$}

'Department of Anesthesiology, Shohada Tajrish hospital, Shahid Beheshti University of Medical Sciences, Tehran, Iran

${ }^{2}$ Functional Neurosurgery Research Center, Shohada Tajrish Comprehensive Neurosurgical Center of Excellence, Shahid Beheshti University of Medical Sciences, Tehran, Iran

\begin{abstract}
Chordomas rare low grade slow-growing malignant tumors with an incidence rate of 1 in 100000 individuals.32\% of chordomas occur in the clivus. Diagnosing Clival chordomas could be challenging due to their rare prevalence, insidious onset, and tendency to involve anywhere throughout the craniospinal column. Treating these tumors can also present as a challenge due to their proximity to vital structures and high recurrence rate. We present a case of massive recurrent Clival chordoma in a 27-year-old patient and discuss the anesthetic considerations in such cases.

Keywords: Clivus Chordoma; Anesthesia Management; Cranio-Spinal Column.
\end{abstract}

*Correspondence to

Faranak Behnaz,

Shohada Tajrish hospital

Shahid Beheshti University of

Medical Sciences, Tehran, Iran

Tel: +982122741174

Email:

faranak.behnaz@gmail.com

Published online 29 June 2019

Citation: Soltanpoor P, Behnaz F, Azizi Faresani H, Seddighi A, Karimian M. Anesthetic considerations in a case with massive recurren clivus chordoma. Int Clin Neurosci J. 2019;6(2):76-78. doi:10.15171/icnj.2019.15.

\section{Introduction}

Chordomas are rare slow-growing low-grade malignant tumors of notochordal origin that often have an insidious onset. ${ }^{1}$ They can occur anywhere along the craniospinal axis. $32 \%$ are intracranial lesions arising extramurally in the clivus and midline structures of the skull base, $32.8 \%$ involve spinal vertebrae, and $29.2 \%$ involve the sacrum. ${ }^{1,2}$ The annual incidence rate is 0.08 to 0.1 per 100000 individuals, and the average age at the time of diagnosis estimated 60 years. Prognosis is poor with a median survival time of 6.29 years and an estimated five-year survival rate of just $67.6 .^{3}$

The clivus is a bony structure of skull base located between the Sella turcica and foramen magnum. Due to its proximity to vital structures such as the brain stem, basilar artery, internal carotid arteries and cranial nerves such as abducens, hypoglossal and vagus nerve treating a tumor in this region can be challenging to treat. ${ }^{3}$ These tumors also present challenges concerning diagnosis due to their rare prevalence, insidious onset, and tendency to involve anywhere throughout the craniospinal axis. ${ }^{1}$ They are also challenging to treat due to their proximity to various vital structures and their high rate of recurrence. ${ }^{1,4}$

Chordomas can have various presentations. Cranial tumors can present with chronic headaches, cranial neuropathies, subarachnoid hemorrhage, cerebrospinal fluid rhinorrhea, and epistaxis. Spinal tumors can cause back pain sphincter dysfunction and paresthesia and weakness in lower extremities. ${ }^{1}$ A multidisciplinary approach including neurosurgery, otolaryngology, anesthesiology, radiology, oncology, and pathology required for successful treatment. ${ }^{3}$

\section{Case Report}

We report a case of clivus chordoma in a 27-year-old male ASA class 1 patient undergoing trans-oral and trans-nasal resection of the tumor with a history of trans-nasal tumor resection ten months before the surgery and 30 sessions of radiotherapy. He presented with headache, dysphagia, and dysarthria. On examination he had paresis of right cranial nerves, including abducens, glossopharyngeal, glossal nerve. The gag reflex was absent.

\section{Head and Neck Examination}

He had adequate mouth opening, normal neck and temporomandibular joint movement and a mallampati class 1 on oropharyngeal examination.

\section{Lab, Imaging, and Histological Findings}

The standard lab test values were within the normal range.

CT scan showed a heterodense area with mass effect on medulla and pons. Expansion to nasopharynx with prominent involvement of clivus, sphenoid, petrous and occipital condyles noted (Figure 1A).

Magnetic resonance imaging showed a heterogenous skull base mass with extension to the nasopharynx and

(c) 2019 The Author(s). This is an open access article distributed under the terms of the Creative Commons Attribution License (http:// creativecommons.org/licenses/by/4.0/), which permits unrestricted use, distribution, and reproduction in any medium, provided the original work is properly cited. 

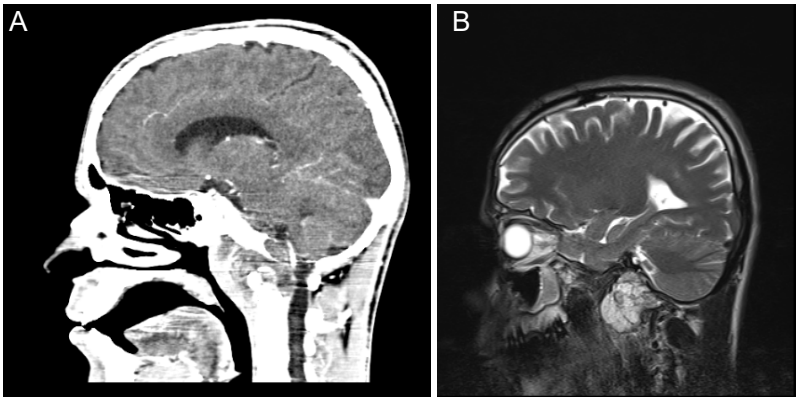

Figure 1. (A) Computed tomography scan shows mass effect on medulla and pons with expansion to nasopharynx. (B)Magnetic resonance imaging shows skull base mass with protrusion to nasopharynx and mass effect on cranial arteries and nerves.

bilateral cerebellopontine angles creating the mass effect on basilar artery consistent with 9, 10 and 12 cranial nerves involvement. (Figure 1B).

Histological results confirmed the diagnosis of chordoma.

The patient was scheduled for combined trans-oral and trans-nasal debulking surgery with the guide of the navigation system under general anesthesia. Due to the importance of having a definite airway and holding to account the fact that the tumor had a massive protrusion in the nasopharynx, we planned for tracheostomy in collaboration with otolaryngology department (Figure 2 ). The procedure was fully explained to the patient and informed written consent obtained.

Pre-induction monitoring included electrocardiogram, heart rate, non-invasive blood pressure, and peripheral oxygen saturation.

Before the induction of anesthesia, a lumbar drain placed in L4-L5 space.

Due to the anticipation of difficult intubation alternative devices such as video-laryngoscope, Airtraq, laryngeal mask airway, tube introducer, and stylet were ready in the operating room.

After the premedication with $2 \mathrm{mg}$ midazolam for anxiolytics and proper pre-oxygenation, general anesthesia induced by administration of $100 \mathrm{mg}$ fentanyl, $100 \mathrm{mg}$ lidocaine, and $250 \mathrm{mg}$ sodium thiopental. Muscle relaxation was achieved using $50 \mathrm{mg}$ atracurium. Endotracheal intubation was performed atraumatically using a cuffed $8 \mathrm{~mm}$ endotracheal tube and correct placement of the tube confirmed by capnography. The maintenance of anesthesia provided with an infusion of $10 \mathrm{mg} / \mathrm{kg} / \mathrm{h}$ of propofol combined with an infusion of 0.1 $\mu \mathrm{g} / \mathrm{kg} / \mathrm{min}$ remifentanil.

Four units of PRBCs were reserved considering the proximity of the tumor to the large cranial arteries and the possibility of sudden massive hemorrhage, and two units of PRBCs were ready for infusion in the operating room. We also placed an arterial catheter for monitoring beat to beat arterial blood pressure and ease of obtaining blood sample throughout the procedure.

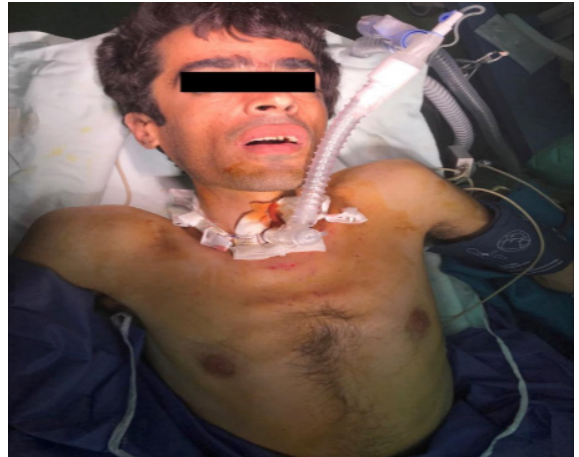

Figure 2. Due to massive protrusion of the tumor to nasopharynx tracheostomy was done to ensure a secure airway.

Tracheostomy has done in collaboration with the otolaryngology department, and the debulking of the tumor performed via trans-oral approach followed by a trans-nasal approach with the guide of the navigation system.

Phenylephrine mesh was used for optimizing the visual field and decreasing the blood loss. Following the application of the phenylephrine mesh, the blood pressure increased for about 15 minutes, which controlled with an additional dose of remifentanil.

The duration of surgery was 6 hours, which was uneventful.

Due to the interruption of the bony structure of the skull base attempting to place nasogastric tube blindly can cause unintentional intracranial tube placement leading to the severe neurologic deficit. Therefore, we placed the nasogastric tube under vision with the aid of video laryngoscope.

The patient was shifted to the neurosurgical ICU and was decannulated on the second postoperative day.

\section{Discussion}

Chordomas are rare primary malignant locally invasive tumors of notochordal origin, which can metastasize to other organs. ${ }^{5}$ They can involve anywhere along with the craniospinal structure.

$32.8 \%$ involve the spine, about $32 \%$ occur intracranially, and $29.2 \%$ involve the sacral bone. The incidence rate is 0.08 per 100000 , which is higher in the male population, 0.1 versus 0.06 in the female population. ${ }^{1}$ Skull base chordomas are even more rare with 1 case in 2,000,000 per year incidence. ${ }^{6}$ Chordomas represent only $0.1 \%$ of tumors in the skull base. ${ }^{7}$

The prognosis of these tumors remains poor with a median survival time of 6.29 years and a 5 -year survival rate of just $67.6 \%{ }^{3}$

Chordomas can divide into three different histopathologic types. Brachyury is a transcription factor expressed in all chordomas and is a particular tumor marker. It can be used to distinguish chordomas from chondrosarcomas, germ cell tumors, and metastatic clear 
cell renal carcinomas. ${ }^{1}$

Treating chordomas can be challenging due to their proximity to various vital structures and their high recurrence rate, therefore, en bloc resection may not be possible, and microsurgery with the aim of gross total resection or subtotal resection followed by adjuvant therapy such as radiotherapy is considered to be the treatment of choice. ${ }^{1,2,4}$

Most chordoma patients treated with the transsphenoidal approach. ${ }^{8}$ However, more extensive tumors treated with the transbasal or transpetrosal approach. ${ }^{2}$ Other approaches include trans mandibular, transcochlear, transcondylar, subtemporal-preauricular, and extreme lateral. ${ }^{1}$ Chordomas are often resistant to conventional radiotherapy.

Imatinib mesylate, a tyrosine kinase inhibitor has been successful in tumor liquefaction, shrinking tumor size and stabilizing tumor growth while decreasing pain and improving life expectancy. ${ }^{1,3}$

Managing the resection of clival chordoma has several anesthetic considerations. Due to the proximity of the tumor to various vital structures, the involvement of multiple subspecialties can be helpful.

Several units of packed red blood cells should be ready to infuse in the operating room in case of sudden massive hemorrhage, and it is optimal to have an arterial line to monitor beat to beat arterial blood pressure.

Holding to account the fact that having a definite airway is imperative, tracheostomy should be considered in case of tumor protrusion to the pharyngeal wall.

Resecting the clival tumor disrupts the integrity of the bony structure of the skull base; therefore, placing the nasogastric tube should be under the vision to avoid the unintentional intracranial placement.

Phenylephrine is a selective alpha-1 adrenergic agonist. It is used in trans-nasal surgery to optimize the visual field and minimize blood loss. It can cause episodes of hypertension and reflex bradycardia, especially in patients with disrupted nasal mucosae due to the history of previous surgery. Using phenylephrine mesh should be in a careful manner, and symptoms should manage accordingly.

\section{Conflict of Interest Disclosures}

The authors declare that they have no conflict of interests.

\section{Ethical Statement}

An informed consent form for publication of the study was obtained for the patient.

\section{References}

1. Khawaja AM, Venkatraman A, Mirza M. Clival chordoma: case report and review of recent developments in surgical and adjuvant treatments. Pol J Radiol. 2017;82:670-675. doi: 10.12659/PJR.902008.

2. Förander $\mathrm{P}$, Bartek J, Fagerlund $\mathrm{M}$, Benmaklouf $\mathrm{H}$, Dodoo E, Shamikh A, Stjärne P, Mathiesen T. Multidisciplinary management of clival chordomas; long-term clinical outcome in a single-institution consecutive series. Acta Neurochir (Wien). 2017;159(10):1857-1868. doi: 10.1007/s00701-0173266-1.

3. Faircloth AC, Noble J. Anesthetic management of a pediatric patient undergoing a pancreatoduodenectomy with portal vein reconstruction. AANA J. 2012;80:37-42.

4. Bhatnagar V, Karmarkar AA, Dwivedi D, Ray A. Anesthetic management of excision of recurrent $\mathrm{C} 2$ chordoma with extension into posterior pharyngeal wall. Journal of Marine Medical Society. 2017;19(2):128.

5. Taran S, Yusof AH, Yusof MI. Endoscopic transoral resection of an axial chordoma: a case report. Malays Orthop J. 2015;9(3):75-7. doi: 10.5704/MOJ.1511.015.

6. Sen C, Triana Al, Berglind N, Godbold J, Shrivastava RK. Clival chordomas: clinical management, results, and complications in 71 patients. J Neurosurg. 2010;113(5):1059-71.

7. Singh N, Bhaskar P, Gupta D, Ambesh SP. Anesthetic management of clival chordoma with retropharyngeal extension: Importance of imaging. Anaesthesia. Pain and Intensive Care. 2013;17(2):211-2.

8. Taniguchi $M$, Kohmura E. Endoscopic endonasal removal of laterally extended clival chordoma using side-viewing scopes. Acta Neurochir (Wien). 2012;154(4):627-32. doi: 10.1007/s00701-011-1225-9. 